\title{
Short term assessment and mitigation of flood risks at river basin level
}

\author{
Z. Boukalová ${ }^{1}$, F. Čejka ${ }^{1} \&$ V. Beneš ${ }^{2}$ \\ ${ }^{1}$ VODNÍ ZDROJE, a.s., Czech Republic \\ ${ }^{2}$ G IMPULS Praha spol. s r.o., Czech Republic
}

\begin{abstract}
This paper deals with an innovation in the area of embankment safety monitoring systems, namely the use of optical fibres and discrete borehole sensors for fulltime monitoring of seepages based on the measurement of temperature, potentially including stress changes in the body of the embankment as well. The paper describes the investigation using the Geophysical Monitoring System (GMS) undertaken by the VODNI ZDROJE Company under the EUREKA project STAMFOR in 2014 followed by the presentation of the strategy of pilot sensors application on various sites in the Czech Republic and abroad planned to be carried out in 2015 .

Keywords: Geophysical Monitoring System (GMS), flood risk assessment, linear terrestrial dikes, levee breach, geotechnical conditions of the embankments, sensors, database, fibre optic.
\end{abstract}

\section{Introduction}

The risk for the population of Europe originating from possible failure of dams and constructions designed for protection against floods (further referred to as embankments) is widespread throughout Europe. In many countries the number of small dams (up to $15 \mathrm{~m}$ of height) significantly exceeds the number of the large ones. For instance, in the UK the number of small dams amounts to about 2,000 in comparison with only a little more than 500 large ones.

Embankments are basic parts of infrastructure, serving for protection of public and economic resources. However, the probability of failure of these embankments increases with time. Extreme hydrogeological events such as heavy rains or sea surges may damage their structure by overflowing, seepages, 
etc. Taking into account the present significant climatic changes, it is therefore essential to bring to the market a technology able to determine the actual conditions of linear water-related constructions (flood embankments, dams, levees, etc.), enabling more effective, faster and safer risk control. In order to achieve sustainable management of water resources, in particular at river basin level, it is of high importance to provide decision-makers with easy access to comprehensive, representative and reliable information on the quality of embankments and dams.

The aim of this innovative approach - that could be conveniently used by stakeholders and river basin managers - is to implement an innovation in embankment safety monitoring, namely the use of optical fibres and borehole sensors for full-time monitoring of embankment seepages. The monitoring is expected to be based on the measurement of temperature and possibly stress changes in optical fibres, caused by seepages and deformations by shifting in the body of the embankment. However, in order to plan properly the installation of the sensors into the embankment, previous geophysical investigation of its structure needs to be carried out. The new monitoring method will be combined with the Geophysical Monitoring System (GMS) developed by Boukalová and Beneš [1] and an online platform for data evaluation and visualisation developed by Boukalová et al. [2]. The aim of GMS is to detect anomalous embankment sections and, based on this investigation, to define the best way of the installation of sensors in different embankment types, namely in the linear flood levees, earth dams and in a limited extent also in rock-fill dams.

\section{First step: GMS}

The GMS methodology provides a low cost solution for the basic tasks and needs in performing maintenance and technical surveillance of the embankments. GMS comprises 3 following basic elements [3]:

- Quick Testing Measurement (QTM):

This phase of the survey is performed by applying the dipole electromagnetic profiling (Slingram) method using a multi-frequency device (i.e. GEM 2). Such a device is able to simultaneously measure apparent resistivity values at several frequencies of the electromagnetic signal; 4 working frequencies are used in GMS by default. The Slingram method is based on the measurement of induction of the primary electromagnetic field of the transmitting coil in the surrounding investigated medium. The primary field induces a secondary field whose intensity depends on the conductivity (resistivity) of the medium surrounding the transmitting coil. In case of GMS the materials forming the body of the embankment and underlying soils and rocks are concerned. The depth to obtain information on the conductivity of the medium depends on the frequency of the primary electromagnetic field.

This testing results in an assessment of inhomogeneous and other potentially unstable segments of the embankment. These segments can also be found by comparing the measured data with data sets from previous measuring campaigns concerning the same object. 
- Diagnostic Measurement (DM):

This phase is used to provide detailed information on the structure of inhomogeneities within the embankment sections highlighted by QTM. The methodology is based on the application of a number of geoelectric methods, in particular the method of electric resistivity tomography, which is often complemented by another method depending on the searched defect type (e.g. self-potential method).

- Measurement of Geomechanical properties (MGM):

The third phase provides complete information on geomechanical properties of the embankment by applying the seismic methods and microgravimetry in combination with the borehole and laboratory tests.

The main benefit of the GMS methodology is a broad utilization of the Slingram method for a quick description of the conditions and monitoring of the embankment.

\subsection{Monitoring function of GMS}

The monitoring function of GMS consists in regularly repeated Slingram measurements preferably under different hydraulic loads. The analysis of a series of repeated measurements brings a new type of geophysical information - it is possible to delimitate levee segments showing time dependent anomalous changes in electric properties of the levee materials. The changes occur most frequently at places of repeated seepage through the levee or underlying materials. In making the analysis, two basic rules have to be respected:

1. It is necessary to differentiate "standard/normal" variations of soil resistivities caused by different moisture content in the levee materials and underlying soils and rocks during repeated measurements. Moisture content fluctuates due to precipitation (climatic conditions) and river water level changes. Such variations of resistivities commonly reach tens to hundreds of percent.

2. Repeated measurements for the assessment of seepage pathways should be performed under contrasting hydrological and hydraulic conditions preferably in the dry period when the reservoir is discharged and the levee is dry (basic measurement) and in high water level conditions or even flood conditions when the reservoir shows maximum water level (control measurement).

An example of the analysis of a pair of repeated measurements is shown in Figure 2. It is the same segment as shown in Figure 1. Curves in the top graph represent resistivities for a frequency of $47025 \mathrm{~Hz}$, measured in the "dry" period (blue curve) and during the flood (red curve). Once the levee is saturated with water during the flood, an overall decline in resistivities is observed which corresponds to "standard/normal" variations. However, local shape variations at the measured curves are what matters. Relative residual resistivity anomalies shown on the bottom graph can help in this case. 


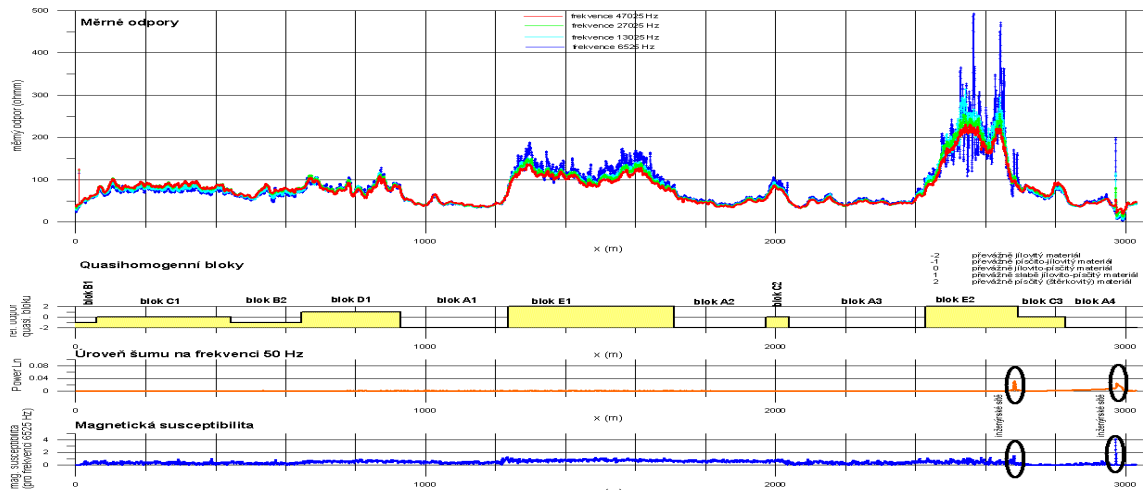

Figure 1: Example of QTM results and interpretation.

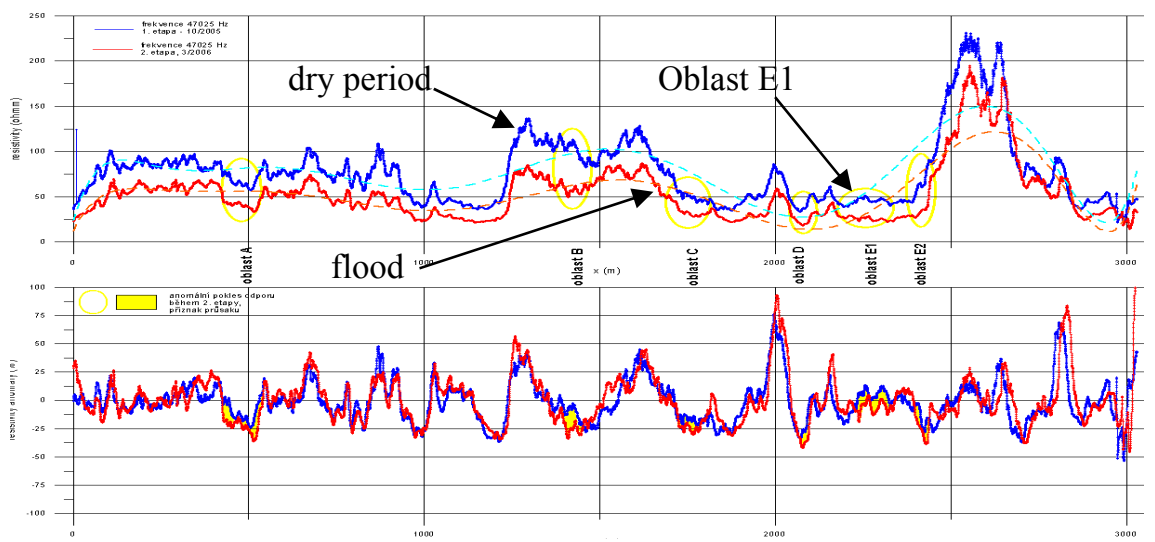

Figure 2: Example of results of repeated measurements and its interpretation.

Those segments where the difference in resistivities between stage 1 and stage 2 reaches 20 and more percent of the value of the regional field (a significant decline of the 'dry period' curve below the 'flood' curve) are considered anomalous. In total, 6 segments were delimited here in this way.

In Figure 2, these segments are highlighted in yellow and marked with letters A through E. On close inspection, fine changes in the shape of resistivity curves in comparison with the surroundings can be observed directly in the measured data as well (top graphs). Nevertheless, in the graphs of residual anomaly these variations are definitely better readable.

Results of the conducted monitoring measurements were verified under flood conditions during visual inspections. In Figure 3, seepage recorded in the anomalous area marked as "oblast E1" is shown. 


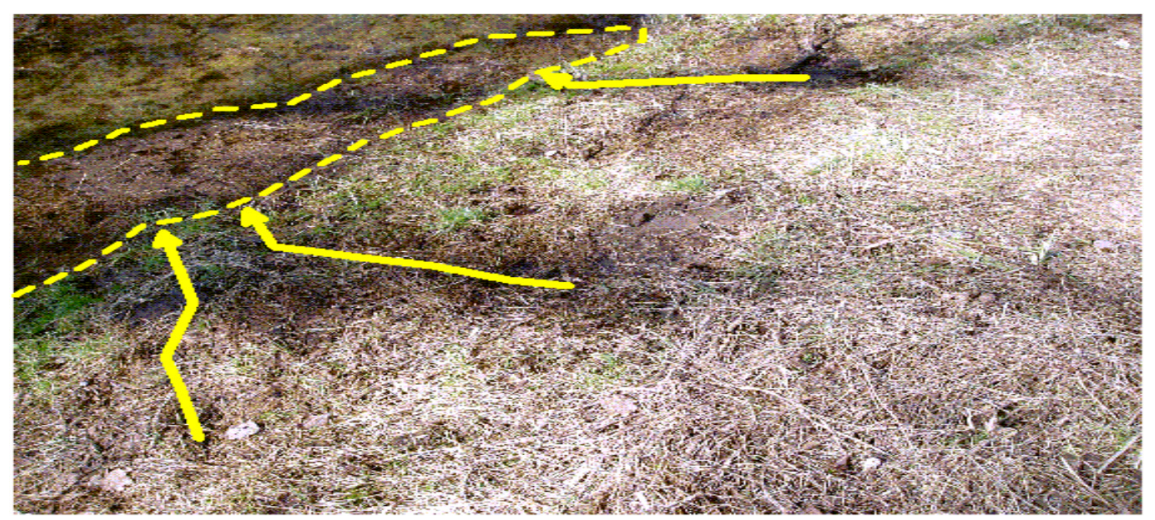

Figure 3: Detail of seepage at the levee base under flood conditions.

\subsection{GMS measurement on selected pilot sites}

The main objective of the geophysical survey performed in 2014 on levees and dams was to provide a general description of their inner structure and homogeneity. The pilot sites for the first stage monitoring were located in the Czech Republic (Sběř reservoir, Petrůvka embankment) and United Kingdom (flood levees in the Humber Estuary).

\subsubsection{Humber estuary}

During the flood event in the Humber estuary, UK - in December 2013 - several levees were significantly damaged by overtopping and subsequent backward erosion. For this reason, this locality proves ideal for the geophysical investigation and following installation of the sensors. For GMS, 15 sites (levee sections) of lengths varying from 500 to $4000 \mathrm{~m}$ have been surveyed in the Humber estuary area; these sites were chosen prior to the survey and further updated by the representatives of the Environment Agency UK with regard to the priorities and time schedule. The results of the geophysical survey will be used:

- to prepare an optimal proposal of the planned geotechnical survey,

- to prepare a plan for repair and maintenance works,

- $\quad$ to prepare an optimal design of the sensor network that could be used for on-line monitoring of the embankments.

The field survey of each site was performed in 2 GMS stages (QTM and DM).

\subsubsection{Sběř and Petrůvka}

The geophysical survey in the Czech Republic - in the village of Petrüvka (the levees protect the village from frequent flash floods on the Petrüvka river) and the Sběr pond - has been performed to assess the structure and homogeneity of the embankments and underlying soils and rocks. The following methods have been used for the assessment: 
- Dipole electromagnetic profiling (Slingram)

- $\quad$ Electric resistivity tomography (ERT)

- $\quad$ Self-potential (SP)

- $\quad$ The results of previous geophysical measurements (year 2011).

The measured data have been evaluated and visualised using the programme DIKINS (3).

\section{Interpretation of the GMS data}

The geophysical investigation performed on the selected pilot sites in 2014 provided substantial information on the inner structure of the embankments. After this information has been combined with the results of the borehole geotechnical survey, the data set will be used to:

- model the critical seepage curve in the body of the embankment

- define appropriate positions for the sensors to be placed

- compare the data measured by different types of sensors

\subsection{Slingram}

The data collected by Slingram have been evaluated using the software DIKINS_Analyzer. In the first stage, the levee segments where measured data had been affected by metallic objects present at/close to the levee (infrastructures, fences, reinforced concrete constructions, etc.) were evaluated. These objects manifest themselves either by a magnetic susceptibility anomaly (metallic objects) or by anomalous data in the function 'Power' (power lines and cables) or by a typical sharp 'bell-shaped' resistivity anomaly (combination of both). The segments at which the above listed anomalies occur are marked in the function as 'Invalid'. This function returns values 0 (segments without significant disturbing effects) or 1 (disturbed segment). The validity of measured data and their interpretation at segments where Invalid $=1$ are reduced. This information can be useful for various purposes, such as:

- $\quad$ Specification of the network positioning

- Assessment of possible illegal networks;

- Localization of construction waste in the body of the levee, etc.

The interpretation of the Slingram measurement continues with a delimitation of the so-called quasihomogeneous blocks (QHBs) within the measured levee (Figure 4). QHBs are levee sections with similar spatial resistivity behaviour, hence similar inner structure and materials used for the construction of the levee can be expected in such a segment. Three basic parameters influence the measured resistivity values in the Humber estuary and need to be taken into account when evaluating the QHBs:

- $\quad$ Material structure (clay, sand, gravel and their mixtures);

- Water content in sediments;

- Ground water salinity. 


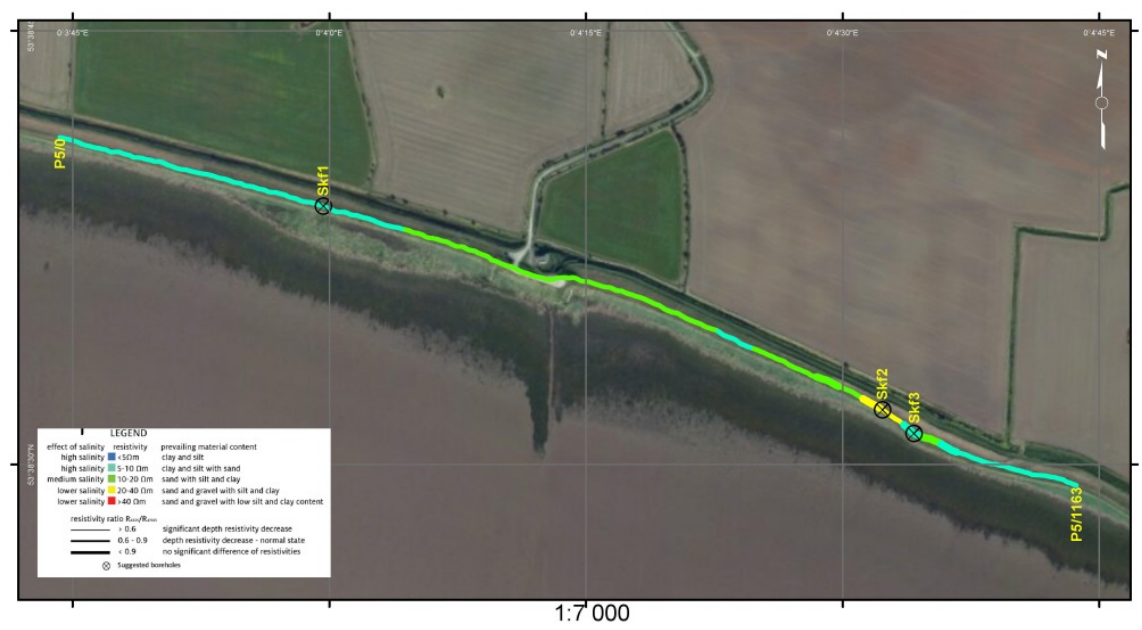

Figure 4: Delimited QHBs on the Skeffling site.

\subsection{Electric Resistivity Tomography (ERT)}

ERT ranks among direct-current geoelectric resistivity methods. The principle of the method is based on the measurement of resistivities of rocks, using a large number of electrodes placed along the profile or in the area. The results of the ERT measurements are interpreted as resistivity models with real spatial resistivity distribution. The models have been computed by the application RES2DInv, widely used software for 2D and 3D inversion of geoelectric data given by resistivity tomography. By way of example, ERT measurements in the Humber Estuary were organized in Schlumberger electrode arrays and the results were obtained using the 3rd iteration of least squares method. According to the resistivity value range at the site, two basic scales $(1-30 \Omega \mathrm{m}$ and $1-150 \Omega \mathrm{m})$ were used. The sections are presented in two depth scale factors (1 and 2) in order to provide more detailed visualisation of the shallow anomalies in the levee (Figure 5).

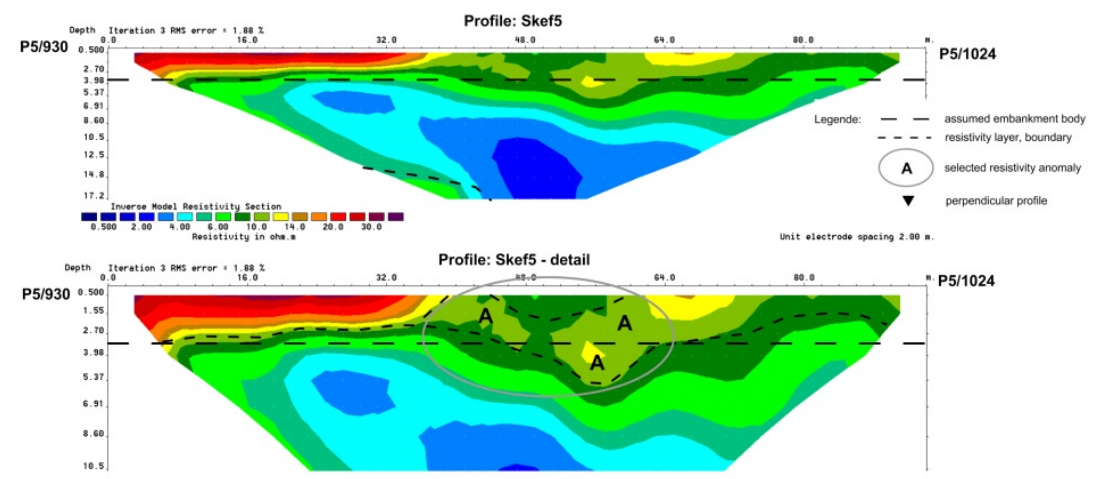

Figure 5: Example of ERT data from the Skeffling site. 
The ERT layouts are not used directly for QHB delimitation, the info is mostly used for detailed diagnosis of the transition structures/anomalies, detailed description of the vertical stratification of the resistivities and validation of the Slingram data.

\subsection{Self-Potential (SP)}

SP method measures the natural electrical potential of the rock environment. In investigating the levees, filtration potential produced by water filtration through a porous medium can be often detected. The principle of this phenomenon consists in unequal mobility of anions and cations transferred by the liquid medium through a porous material. This inequality generates usually measurable negative potential at the point of infiltration and positive potential at the point of outflow. This theory of course assumes the existence of active seepage, i.e. water must be present on the water-facing side of the levee; the SP method cannot be used in case of flood levees that are dry for a long period.

The outcomes of the measurements using the SP method are profile curves graphs of electric potentials. Based on their analysis, the locations of potential seepage through the levee can be identified. In the levees, especially the local minima of SP curves (water infiltration and seepage pathway) are considered to be risk posing anomalies. Local maxima in the direction from the landside toe of the levee can be interpreted as seepage outflows. This method is also suitable for repeated monitoring measurements aiming to monitor long-term changes in the seepage regime of the levee and underlying materials.

\section{Second step: sensing systems}

Taking into account recent development in the field of sensing technologies, 2 different types and arrangements of sensors will be considered within this project.

\subsection{Vertical borehole discrete sensors}

These sensors will be installed at cross section profiles on the locations predefined based on the data from the GMS, and geotechnical and modelling investigations. These sensors will provide (a) the data necessary for independent control of the measurements made by the distributed sensors (see below) and (b) the calibration data for the model. The application of this discrete system will be considered for sites with rare occurrence of defects and/or low budget installation. The discrete sensor systems are planned to include sets of thermometers and piezometers.

\subsection{Linear distributed sensors}

This monitoring system will provide data based on the measurement of changes in temperature by optical fibres (OF) placed along the land toe of the embankment. The recent OF temperature measurement systems are able to provide sufficiently reliable data of up to several kilometres of fibre length, thus providing information on possible risk-posing changes occurring in the body of a 
significantly long embankment section. Additionally, a system allowing indirect identification of flow rate in the subsoil layer of interest is planned to be used.

The measurement of temperature along the installed optical fibre will be made using optical time domain reflectometry, namely by analysing the Raman and possibly also Brillouin light scattering.

The draft design of the configuration of sensors is provided in Figure 6.

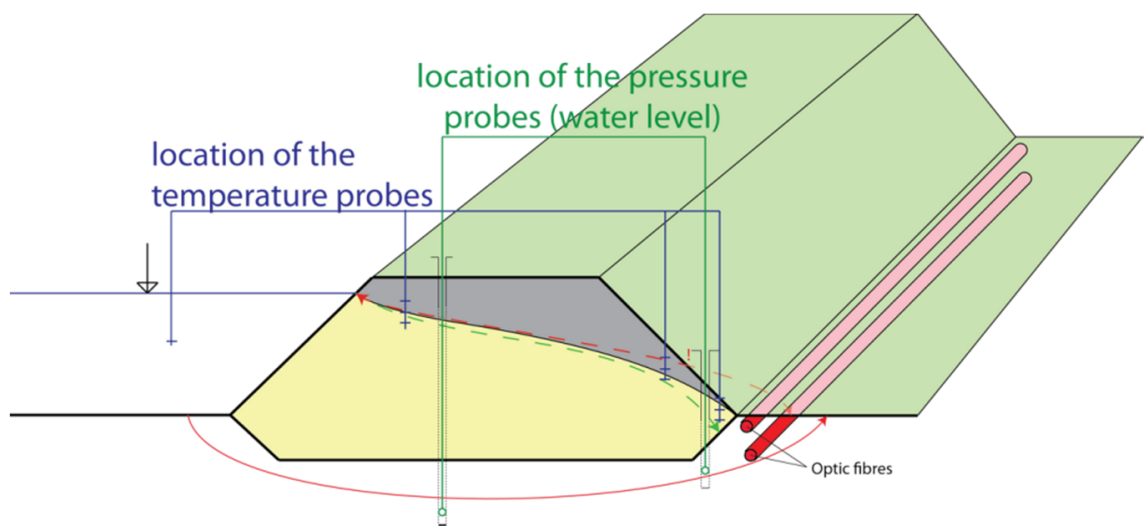

Figure 6: General idea of the sensor configuration.

\section{Third step: online platform}

The last objective of the STAMFOR project is to develop an online platform that will serve as an autonomous system for data gathering, evaluation and visualisation from the GMS, modelling and sensing systems. In addition to that, the platform will be enhanced by an early warning system providing a direct GSM and/or internet messaging service.

The draft flow chart of the online platform is given in Figure 7.

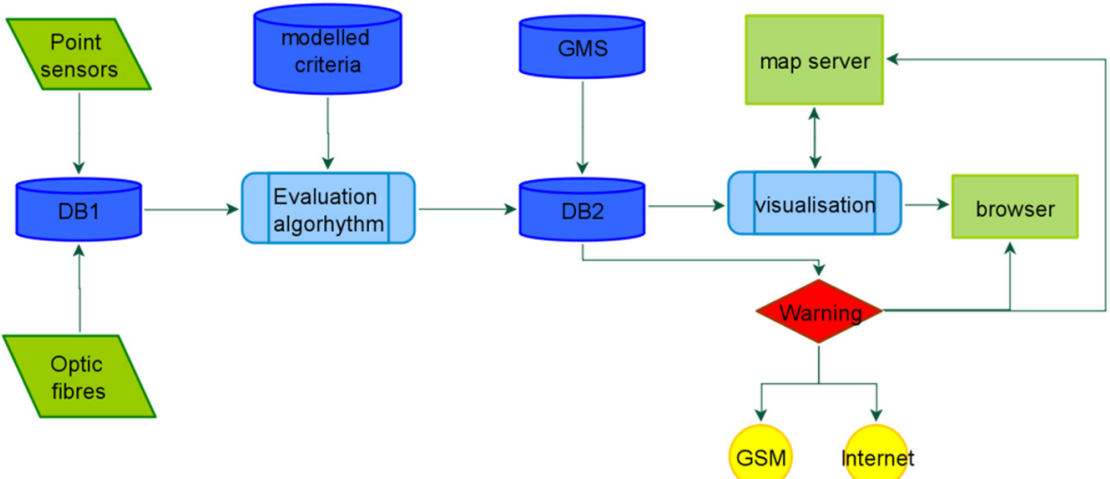

Figure 7: Online platform flow chart. 


\section{Conclusions}

STAMFOR is developing an embankment stability and risk assessment system that can be used by local stakeholders and levee managers. It comprises a toolkit for assessing the material structure of embankments (GMS), in situ sensing systems and an online service. The monitoring kit will deliver all information necessary for a calculation of the inner composition of a damming structure while the sensor systems will measure the actual parameters related to loading. The service will determine the risk of flooding from a combination of the strength of a damming structure and its loading. It allows assessing the acute risk of flooding and taking appropriate action, such as reinforcement of the embankments or installation of a permanent monitoring system. Smart sensors facilitate real-time monitoring and evaluation of embankment performance and generate data that contribute to a better understanding of their state.

The combination of GMS with sensors and the online platform will allow disseminating these sophisticated technologies to institutions that are responsible for the protective function of levees and dams. The technology provides nonexperts with measuring instruments by means of which they can obtain data concerning the dam structure. Furthermore, non-experts will be able to place sensors in embankments that will provide real time information on the hydrological conditions in relation to the strength of the embankment. It is this combination of existing measuring systems (GMS and sensors) with an advanced online visualisation platform that is highly innovative and addresses the needs of river basin managers and local water administrative bodies all over the world.

\section{Acknowledgements}

This paper was written thanks to the E!8688 EUREKA project STAMFOR, financed by the Ministry of Education, Youth and Sports, Czech Republic and the company VODNÍ ZDROJE, a.s.

\section{References}

[1] Boukalová, Z. \& Beneš, V., Dike breaks prevention as the process of flooding protection. Proc. of the IAHR Congress: Venice, Italy, 2007.

[2] Boukalová, Z., Beneš, V., Čejka, F. \& Veselý, L., Development of modular system for application in the Integrated Water Management practices and floods prevention, organization of the national/international eGMS database. Proc. of the, FLOODrisk 2012 conference; Rotterdam, Netherlands, 2012.

[3] Boukalová, Z. \& Beneš, V., Application of GMS System in the Czech Republic - Practical Use of IMPACT, FLOODSite and GEMSTONE Projects Outcomes. Proc. of the FLOODrisk 2008 conference; Oxford, UK, 2008. 